\title{
Switching from oxycodone to methadone in advanced cancer patients
}

\author{
Sebastiano Mercadante • Patrizia Ferrera • \\ Patrizia Villari • Claudio Adile • Alessandra Casuccio
}

Received: 9 March 2011 / Accepted: 24 August 2011 /Published online: 7 September 2011

(C) Springer-Verlag 2011

\begin{abstract}
Purpose The aim of this study was to prospectively evaluate the outcomes and the conversion ratio of switching from oxycodone to methadone in advanced cancer patients admitted to an acute palliative care unit.

Patients and methods A prospective study was carried out on a cohort of consecutive sample of patients receiving oxycodone, who were switched for different reasons mainly because of an inconvenient balance between analgesia and adverse effects. An initial conversion ratio between oxycodone and methadone was 3.3:1. Intensity of pain and symptoms associated with opioid therapy were recorded, and a distress score (DS) was also calculated as a sum of symptom intensity. A successful switching was considered when the intensity of pain and/or DS or the principal symptom requiring switching decreased at least of 33\% of the value recorded before switching.

Results Nineteen out of 542 patients admitted to the unit in 1 year underwent a switching from oxycodone to methadone.
\end{abstract}

S. Mercadante $(\bowtie) \cdot$ P. Ferrera $\cdot$ P. Villari $\cdot$ C. Adile

Pain Relief and Palliative Care Unit,

La Maddalena Cancer Center,

Via San Lorenzo 312,

90146 Palermo, Italy

e-mail: terapiadeldolore@lamaddalenanet.it

S. Mercadante

e-mail: 03sebelle@gmail.com

S. Mercadante

Palliative Medicine, University of Palermo,

Palermo, Italy

A. Casuccio

Department of Experimental Biomedicine and Clinical

Neuroscience, University of Palermo,

Palermo, Italy
Almost all substitutions were successful. The prevalent indication for opioid switching was uncontrolled pain and adverse effects (12 patients). No significant changes between the initial conversion ratio and final conversion ratio between the two opioids were found.

Conclusion Switching from oxycodone to methadone is a reliable method to improve the opioid response in advanced cancer patients. A ratio of 3.3 appears to be reliable, even at high doses. Further studies should be performed to confirm these results in other settings and with very high doses of oxycodone.

Keywords Oxycodone $\cdot$ Methadone $\cdot$ Opioid switching · Conversion ratio

\section{Introduction}

Oxycodone has been shown to be as versatile and flexible as oral morphine in the management of cancer pain. Controlled-release oxycodone is widely accepted as an alternative to morphine, resulting as safe and effective as controlled-release morphine [11], and is the preferred drug for switching from morphine in UK [12]. Oxycodone was efficacious and well tolerated as a first-line opioid in doses of $20-40 \mathrm{mg} /$ day after 3 weeks [14]. The use of relatively high doses of oxycodone in terminal cancer patients was found to be safe, efficient, and unrelated to shorter survival times $[1,7]$.

Opioid substitution has been found to produce an improvement in the opioid response [3]. Given the increasing use of oxycodone, even at high doses, it is likely that some patients may not respond and require an opioid switching. Previous studies have shown outcomes and conversion ratios between morphine, hydromorphone, 
and transdermal fentanyl [8]. However, switching from oxycodone to methadone has never been assessed and an indirect conversion ratio with morphine has been used in some circumstances [9]. The aim of this study was to assess the outcome and the final conversion ratio between oxycodone and methadone.

\section{Patients and methods}

A prospective study was carried out in a sample of consecutive patients admitted to an acute palliative care unit for a period of 1 year. Informed consent (from relatives in case of cognitive failure) and institutional approval were obtained. Advanced cancer patients receiving oxycodone who were required to switch opioid therapy were included in four categories:

(a) Patients presenting relevant adverse effects despite good pain control; to be switched, patients had to present at least one relevant symptom, such as drowsiness, confusion, or myoclonus with intensity= 2 on a scale from 0 to 3 (see below), or other symptoms (constipation, dry mouth) rated as severe

(b) Patients with a poor analgesic response despite having their dose doubled in 1 week

(c) Patients with both poor pain control and prevalent adverse effects

(d) Patients who were switched for patient's preference and/or convenience (example dysphagia limiting the oral route) or other reasons (renal failure)

The scenario was that of a clinical setting of an intensive acute palliative care unit. Intravenous methadone was used when the oral tract was unavailable. Rescue doses of methadone were used, intravenously or orally, using the equivalent of one sixth of the daily dose, to support the switching. According to previous experience, based on an indirect conversion from oral morphine to oral methadone [9], the initial conversion ratio was oral methadone $20=$ intravenous methadone $16=$ oral oxycodone 70 (oral oxycodone-oral methadone ratio approximately $3.5: 1$ ). According a "stop and go" approach previously described with morphine, oxycodone was stopped and methadone was given immediately, being the daily dose calculated divided in three administrations [10]. After the initial dose, the subsequent doses were flexible and were changed timely to fit the patients' needs in an attempt to find the best balance between pain and opioid-related symptoms.

Adjuvant drugs, previously administered to control symptoms due to illness or treatment, were continued at the same doses during the switching. Non-opioid analgesics were also continued if previously administered at the same doses. No patient received anticancer therapy during the course of the study. All patients were strictly monitored by a team consisting of doctors and nurses experienced in palliative care. In particular, pain and symptoms are recorded in a sheet by nurses four times a day, as a routine, and four rounds or more are performed by the team to explore clinical changes. A physician on duty and a team component on call were available. Daily doses were changed, according to the amount of drugs consumed as rescue doses in the previous day and the clinical judgment, to achieve the best clinical balance between analgesia and adverse effects.

The following data were recorded:

- Age, gender, primary cancer, and performance status

- Pain intensity, measured using the patient's self report on a numerical $0-10$ scale

- Symptoms associated with opioid therapy or commonly present in advanced cancer patients, such as nausea and vomiting, drowsiness, confusion, constipation, dry mouth, myoclonus, sweating, using a scale from 0 to 3 , corresponding to a verbal scale (not at all, slight, a lot, awful), were recorded. A distress score (DS) was also calculated as a sum of symptom intensity. Although never validated, this score has been previously used in different studies for determining the "weight" of adverse effects. The aim of using a sum of intensities is justified by the high variability of symptom intensity requiring opioid substitution in individual patients. This score is able to determine a general improvement of symptoms. The evaluation of the changes in the intensity of a single symptom, different for each patient, makes a global evaluation and statistics for a group of patients, practically impossible. Moreover, it is not rare to switch a patient for more than one symptom. Changes in DS have been already used to assess outcomes of switching [9]. On the other hand, an important decrease of the principal symptom which required the switching is another parameter to take into consideration. Thus, these

Table 1 Characteristics of patients

\begin{tabular}{lc}
\hline Gender (m/f) & $13 / 4$ \\
Age (years) & $65.9(9.1)$ \\
& $\quad$ Cancer diagnosis \\
Lung & 7 \\
Breast & 3 \\
Prostate & 2 \\
Others & 7 \\
Karnofsky status & $46.5(4.9)$ \\
Time from switching to hospital discharge (days) & $4.7(1.7)$ \\
Time to achieve stabilization (days) & $3.4(1.8)$ \\
\hline
\end{tabular}

Mean values $( \pm \mathrm{SD})$ 
Table 2 Data of patients who completed the switching from oxycodone to methadone successfully at the different intervals considered

\begin{tabular}{lccccc}
\hline & $T_{0}$ & $T(24 \mathrm{~h})$ & $T(48 \mathrm{~h})$ & $T(72 \mathrm{~h})$ & $T$ end \\
\hline Oxycodone & $305.9(249.1)$ & & & \\
Methadone & $86.1(78.0)$ & $91.8(88.5)$ & $88.0(81.2)$ & $93.8(89.2)$ & $88.6(77.8)$ \\
Pain & $6.6(1.7)$ & $3.7(1.2)^{*}, * *$ & $2.8(1.3)^{*}, * *$ & $2.8(1.2)^{*}, * *$ & $2.6(1.0)^{*}, * *$ \\
DS & $4.5(1.2)$ & $3.1(1.2)^{* * *}$ & $2.9(1.0)^{* * *}$ & $2.9(0.7)^{* * *}$ & $2.7(1.0)^{* * *}$ \\
\hline
\end{tabular}

Data are expressed as a mean $( \pm \mathrm{SD})$

$D S$ distress score

${ }^{*} p<0.0005$ vs $T_{0} ; * * p=0.005$ vs $24 \mathrm{~h} ; * * * p=0.001$ vs $T_{0}$

parameters were used to define a successful switching (see above). Symptoms were assessed by the patient, whenever possible. However, in patients who had severe cognitive failure, a proxy evaluation was taken into account.

The following parameters and intervals were recorded: daily opioid doses, pain intensity, and DS before switching $\left(T_{0}\right)$, at 24-h intervals for 3 days, and at time of stabilization which is the time to reach a stable daily dose considered as the first of two consecutive days requiring no more than two rescue doses ( $T$ end) and time from admission to hospital discharge ( $T$ discharge). Data were analyzed using the paired Wilcoxon signed-rank test to compare opioid dosage, pain intensity scores, and symptom intensity scores in the different time intervals. Spearman's rho correlation coefficient was calculated to measure the strength of the relationship between the previous dose of oxycodone and the methadone dosage at discharge. All $p$ values were two sided, and $p$ values less than 0.05 were considered to indicate statistical significance.

\section{Results}

Nineteen out of 542 patients admitted to the unit in 1 year underwent a switching from oxycodone to methadone, and one patient was switched from methadone to oxycodone (not considered for analysis). The characteristics of patients are presented in Table 1. Almost all substitutions were successful. One patient was discharged before achieving adequate stabilization on request of the relatives. One patient could not appropriately be assessed because he was severely ill and required palliative sedation before dying. The indications for opioid switching were uncontrolled pain and adverse effects (12 patients), adverse effects ( 3 patients), uncontrolled pain ( 3 patients), and renal failure $(n=1)$.

No significant changes between the initial conversion ratio and final conversion ratio between the two opioids were found (see Table 2). A significant correlation between oxycodone doses and final methadone doses was found (Spearman's rho correlation coefficient $=0.945 ; p<0.01$ ), but not between oxycodone doses and final conversion ratio (see Fig. 1).

\section{Discussion}

In this study, the switching from oxycodone to methadone was successful in the majority of patients. The indirect conversion ratio used to start the daily dose of methadone did not change, confirming the reliability of the ratio chosen. Of interest, the higher oxycodone dose, the higher methadone dose was found, with a correlation between opioid doses. On the other hand, no correlation between oxycodone doses and final conversion ratio was found.

The most controversial drug for opioid switching is methadone, and the vast majority of studies focused on switching from different opioids to methadone. The advantages of using methadone for refractory pain or adverse effects with other opioids have been invariably reported in literature [8]. The absence of known metabolites allowing a safe use with renal failure, extra-opioid activity on NMDA receptor system, and low cost are the positive

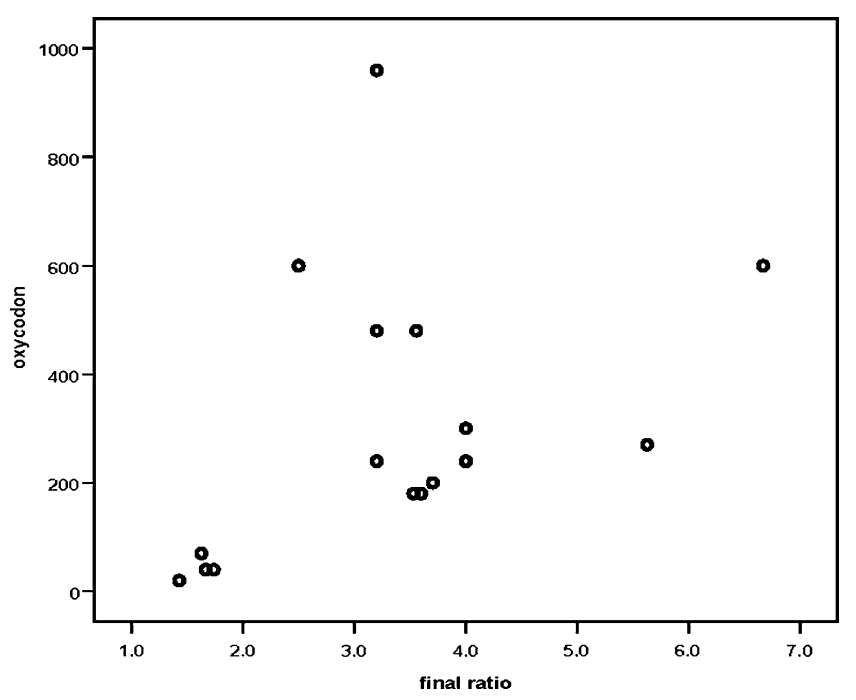

Fig. 1 Plot of oxycodone doses and final conversion ratio 
characteristics of this agent. However, methadone has major pharmacokinetic and pharmacodynamic differences capable of making the switching more complex. Specifically, the lack of reliable equianalgesic conversion ratios, increased potency associated with methadone in patients previously exposed to high-dose opioids, large interindividual variability in methadone pharmacokinetics, and the potential for pharmacological interaction with other drugs have made the clinical use of methadone difficult.

Systematic reviews on switching to methadone have shown a large heterogeneity in terms of opioid ratios, outcomes, and modality of switching utilized $[4,5,10,15$, 16]. The dose of methadone cannot be easily predicted as it will depend on a series of factors, including individual response, pharmacodynamics, pain mechanism, pharmacogenetics, and degree of cross-tolerance [4].

Data regarding the switching from oxycodone are lacking, and conversion ratios have never been assessed in specific studies, unless one utilizes indirect conversion ratios through morphine equivalents [9]. In this previous survey, an approximate ratio of 3.3 was found. These data were confirmed in this selective study on opioid switching from oxycodone to methadone, differently from studies of morphine-methadone switching, where there was a correlation between the previous dose of morphine and the final ratio, particularly in patients receiving high doses of morphine $[2,6,13]$. In this study, the doses of oxycodone were in the medium range of clinical dosages (extremes 20$960 \mathrm{mg} /$ day), so that this approach should be better assessed in patients receiving very high doses of oxycodone. In this study, seven patients were receiving doses of $\geq 300 \mathrm{mg} /$ day, but the conversion ratio was not different from patients receiving lower doses, although the number of patients cannot be considered sufficient to provide definitive data. The principal limitation is represented by the low number of patients included in this study, which however, represent a discrete amount of patients recruited in an acute palliative care unit during a 1-year period, where a consistent number of opioid substitutions are performed [9].

In conclusion, switching from oxycodone to methadone is a reliable method to improve the opioid response in advanced cancer patients. A ratio of 3.3 appears to be reliable even at relatively high doses. However, these data were obtained in an intensive palliative care unit, with a high level of monitoring, and should be considered as preliminary. Further studies should be performed to confirm these results in other settings and with very high doses of oxycodone.
Conflicts of interest There is no conflict of interest.

\section{References}

1. Bercovitch M, Adunsky A (2006) High dose controlled-release oxycodone in hospice care. J Pain Palliat Care Pharm 20:33-39

2. Bruera E, Pereira J, Watanabe S et al (1996) Opioid rotation in patients with cancer pain. A retrospective comparison of dose ratios between methadone, hydromorphone, and morphine. Cancer 78:852-857

3. Expert Working Group of the European Association of Palliative Care Network, Cherny N, Ripamonti C, Pereira J, Davis C, Fallon M, McQuay H, Mercadante S, Pasternak G, Ventafridda V (2001) Strategies to manage the adverse effects of oral morphine: an evidence-based report. J Clin Oncol 19:2542-2554

4. Fine PG, Portenoy RK (2009) Establishing best practices for opioid rotation: conclusions of an expert panel. J Pain Symptom Manage 38:418-425

5. Knotkova H, Fine P, Portenoy RK (2009) Opioid rotation: the science and the limitations of the equianalgesic dose table. J Pain Symptom Manage 38:426-439

6. Lawlor P, Turner K, Hanson J, Bruera E (1998) Dose ratio between morphine and methadone in patients with cancer pain: a retrospective study. Cancer 82:1167-1173

7. Mercadante S, Ferrera P, David F, Casuccio A (2011) The use of high doses of oxycodone in an acute palliative care unit. Am J Hosp Pall Care 28:242-244

8. Mercadante S, Bruera E (2006) Opioid switching: a systematic and critical review. Cancer Treat Rev 32:304-315

9. Mercadante S, Ferrera P, Villari P, Casuccio A, Intravaia G, Mangione S (2009) Frequency, indications, outcomes, and predictive factors of opioid switching in an acute palliative care unit. J Pain Symptom Manage 37:632-641

10. Mercadante S, Casuccio A, Calderone L (1999) Rapid switching from morphine to methadone in cancer patients with poor response to morphine. J Clin Oncol 17:3307-3312

11. Reid CM, Martin RM, Sterne JA, Davies AN, Hanks GW (2006) Oxycodone for cancer-related pain: meta-analysis of randomized controlled trials. Arch Intern Med 166:837-843

12. Riley J, Ross JR, Rutter D, Wells AU, Goller K, du Bois R, Welsh K (2006) No pain relief from morphine? Individual variation in sensitivity to morphine and the need to switch to an alternative opioid in cancer patients. Support Care Cancer 14:56-64

13. Ripamonti C, Groff L, Brunelli C et al (1998) Switching from morphine to oral methadone in treating cancer pain: what is the equianalgesic dose ratio? J Clin Oncol 16:3216-3221

14. Silvestri B, Bandieri E, Del prete S et al (2008) Oxycodone controlled-release as first choice therapy for moderate-to-severe cancer pain in Italian patients. Clin Drug Invest 28:399-407

15. Slatkin N (2009) Opioid switching and rotation in primary care: implementation and clinical utility. Curr Med Res Opin 25:21332159

16. Weschules D, Bain K (2008) A systematic review of opioid conversion ratios used with methadone for the treatment of pain. Pain Med 9:595-612 